International Journal of Modern Physics B

(C) World Scientific Publishing Company

\title{
Ground state of many-body lattice systems via a central limit theorem
}

\author{
CARLO PRESILLA \\ Dipartimento di Fisica, Università di Roma "La Sapienza", \\ Piazzale A. Moro 2, Roma 00185, Italy \\ Center for Statistical Mechanics and Complexity, Istituto Nazionale per la Fisica della Materia, \\ Unità di Roma 1, Roma 00185, Italy \\ Istituto Nazionale di Fisica Nucleare, Sezione di Roma 1, Roma 00185, Italy \\ MASSIMO OSTILLI \\ Dipartimento di Fisica, Università di Roma "La Sapienza", \\ Piazzale A. Moro 2, Roma 00185, Italy \\ Center for Statistical Mechanics and Complexity, Istituto Nazionale per la Fisica della Materia, \\ Unità di Roma 1, Roma 00185, Italy \\ Received DAY MONTH YEAR \\ Revised DAY MONTH YEAR
}

\begin{abstract}
We review a novel approach to evaluate the ground-state properties of many-body lattice systems based on an exact probabilistic representation of the dynamics and its long time approximation via a central limit theorem. The choice of the asymptotic density probability used in the calculation is discussed in detail.
\end{abstract}

Keywords: Lattice quantum models; Probability theory; Stochastic processes

\section{Introduction}

The real- or imaginary-time dynamics of systems described by a finite Hamiltonian matrix, representing bosonic or fermionic degrees of freedom, admits an exact probabilistic representation in terms of a proper collection of independent Poisson processes ${ }^{1,2,3}$. For a lattice system, the Poisson processes are associated to the links of the lattice and the probabilistic representation leads to an optimal algorithm ${ }^{3}$ which coincides with the Green Function Quantum Monte Carlo method in the limit when the latter becomes exact ${ }^{4}$.

In the recent Ref. 5 we have exploited the above probabilistic representation to derive analytical expressions for the matrix elements of the evolution operator in the long time limit. In this way, the ground-state energy as well as the expectation of a generic operator in the ground state of a lattice system without sign problem are obtained as the solution of a simple scalar equation. The result is based on the application of a central limit theorem to the rescaled multiplicities of the values assumed by the potential and hopping energies in the configurations dynamically visited by the system. As a consequence, the probabilistic expectations can be cal- 
culated by using a Gaussian-like probability density. In this paper, we briefly review the approach developed in Ref. 5 and discuss in detail the choice of the asymptotic probability density used in the calculation.

\section{Exact probabilistic representation of lattice dynamics}

We illustrate our approach in the case of imaginary-time dynamics for a system of hard-core bosons described by the Hamiltonian

$$
\hat{H}=-\sum_{i \neq j \in \Lambda} \sum_{\sigma=\uparrow \downarrow} \eta_{i j} c_{i \sigma}^{\dagger} c_{j \sigma}+\hat{V}
$$

where $\Lambda \subset Z^{d}$ is a finite $d$-dimensional lattice with $|\Lambda|$ sites and $c_{i \sigma}$ the commuting destruction operators at site $i$ and spin index $\sigma$ with the property $c_{i \sigma}^{2}=0$. The potential operator $\hat{V}$ is arbitrary, e.g. for the Hubbard model $\hat{V}=\sum_{i \in \Lambda} \gamma_{i} c_{i \uparrow}^{\dagger} c_{i \uparrow} c_{i \downarrow}^{\dagger} c_{i \downarrow}$. For simplicity, we assume $\eta_{i j}=\epsilon$ if $i$ and $j$ are first neighbors and $\eta_{i j}=0$ otherwise.

In order to study the ground-state properties of the Hamiltonian $\hat{H}$ it is sufficient to evaluate the long time behavior of $\sum_{\boldsymbol{n}}\left\langle\boldsymbol{n}\left|e^{-\hat{H} t}\right| \boldsymbol{n}_{0}\right\rangle$, where $\boldsymbol{n}=$ $\left(n_{1 \uparrow}, n_{1 \downarrow}, \ldots, n_{|\Lambda| \uparrow}, n_{|\Lambda| \downarrow}\right)$ are the lattice occupation numbers taking the values 0 or 1. In fact, the ground-state energy is given by

$$
E_{0}=\lim _{t \rightarrow \infty}-\partial_{t} \log \sum_{\boldsymbol{n}}\left\langle\boldsymbol{n}\left|e^{-\hat{H} t}\right| \boldsymbol{n}_{0}\right\rangle,
$$

while the quantum expectation of a generic operator $\hat{O}$ in the ground state of $\hat{H}$ can be obtained via the Hellman-Feynman theorem ${ }^{5}$ by evaluating the ground-state energy $E_{0}(\xi)$ of the modified Hamiltonian $\hat{H}+\xi \hat{O}$.

At any finite time $t$, the matrix elements of the evolution operator considered above admit the exact probabilistic representation

$$
\sum_{\boldsymbol{n}}\left\langle\boldsymbol{n}\left|e^{-\hat{H} t}\right| \boldsymbol{n}_{0}\right\rangle=\mathrm{E}\left(\mathcal{M}_{\boldsymbol{n}_{0}}^{t}\right)
$$

where $\mathcal{M}_{\boldsymbol{n}_{0}}^{t}$ is a stochastic functional defined in terms of independent Poisson processes associated to the links of the lattice, see Ref. 3 for a detailed description. At each jump of a Poisson process relating sites $i$ and $j$ with spin $\sigma$ and taking place at a given configuration $\boldsymbol{n}$, a particle of spin $\sigma$ moves from site $i$ to site $j$ or vice versa if the mod 2 sum of the occupations of these two sites is $\lambda_{i j \sigma}(\boldsymbol{n})=1$, while the lattice configuration $\boldsymbol{n}$ remains unchanged if $\lambda_{i j \sigma}(\boldsymbol{n})=0$. Hereafter, links with $\lambda_{i j \sigma}=1$ will be called active. By ordering the jumps according to the times $s_{k}$, $k=1, \ldots, N_{t}$, at which they take place in the interval $[0, t)$, we define a trajectory as the Markov chain $\boldsymbol{n}_{1}, \boldsymbol{n}_{2}, \ldots, \boldsymbol{n}_{N_{t}}$ generated from the initial configuration $\boldsymbol{n}_{0}$. The number of jumps $N_{t}$ is, of course, a random integer associated to each trajectory. We associate to each trajectory also two sequences, $A_{0}, A_{1}, \ldots, A_{N_{t}-1}$ and $V_{0}, V_{1} \ldots, V_{N_{t}}$, representing the number of active links and the potential energy of the visited configurations

$$
A_{k}=\sum_{(i, j) \in \Gamma} \sum_{\sigma=\uparrow \downarrow} \lambda_{i j \sigma}\left(\boldsymbol{n}_{k}\right),
$$




$$
V_{k}=\left\langle\boldsymbol{n}_{k}|\hat{H}| \boldsymbol{n}_{k}\right\rangle=\left\langle\boldsymbol{n}_{k}|\hat{V}| \boldsymbol{n}_{k}\right\rangle .
$$

Here, $\Gamma$ is the set of system links, i.e. the pairs $(i, j)$ with $i<j$ and $i, j \in \Lambda$ such that $\eta_{i j} \neq 0$. The stochastic functional $\mathcal{M}_{n_{0}}^{t}$ which appears in Eq. (3) actually depends on the jump times $s_{1}, s_{2}, \ldots, s_{N_{t}}$ and on the corresponding sequences $A_{0}, A_{1}, \ldots, A_{N_{t}-1}$ and $V_{0}, V_{1} \ldots, V_{N_{t}}$.

\section{Probabilistic expectation in the long time limit}

Evaluating the expectation $\mathrm{E}\left(\mathcal{M}_{\boldsymbol{n}_{0}}^{t}\right)$ over the detailed sequences above specified can be done numerically by a Monte Carlo method ${ }^{3}$. In Ref. 5 we have demonstrated that an analytical expression of $\mathrm{E}\left(\mathcal{M}_{\boldsymbol{n}_{0}}^{t}\right)$ can be obtained in the limit of long times. This result is reached in four steps described in the next subsections. The crucial point is that, if one integrates over all the possible jumps times, what matter are not the detailed sequences $A_{0}, A_{1}, \ldots, A_{N_{t}-1}$ and $V_{0}, V_{1} \ldots, V_{N_{t}}$ but the multiplicities $N_{A}$ and $N_{V}$ of the possibles values which the variables $A$ and $V$ may assume. We call $\mathscr{A}$ and $\mathscr{V}$ the sets of these values and $m_{\mathscr{A}}$ and $m_{\mathscr{V}}$ their cardinalities. It is clear that the nature of these sets depends only on the structure of the system Hamiltonian, not on the values of the Hamiltonian parameters. The expectation $\mathrm{E}\left(\mathcal{M}_{\boldsymbol{n}_{0}}^{t}\right)$ is reduced to an average over $N_{A}$ and $N_{V}$. For $t \rightarrow \infty$, this average can be evaluated analytically by using saddle-point techniques and a central limit theorem.

\subsection{Canonical decomposition of the expectation}

Referring to Ref. 5 for the details, we decompose the expectation as a series of conditional expectations with a fixed number of jumps (canonical averages)

$$
\mathrm{E}\left(\mathcal{M}_{\boldsymbol{n}_{0}}^{t}\right)=\sum_{N=0}^{\infty} \mathrm{E}\left(\mathcal{M}_{\boldsymbol{n}_{0}}^{t} \mid N_{t}=N\right) .
$$

Integrating over the $N$ jumps times, each term of the series (6) can be written as

$$
\mathrm{E}\left(\mathcal{M}_{n_{0}}^{t} \mid N_{t}=N\right)=\left\langle\mathcal{W}_{N}(t) \prod_{A \in \mathscr{A}} A^{N_{A}}\right\rangle_{N},
$$

where $\langle\cdot\rangle_{N}$ means average over the trajectories with $N$ jumps generated by extracting with uniform probability one of the active links available at the configurations $\boldsymbol{n}_{0}, \boldsymbol{n}_{1}, \ldots, \boldsymbol{n}_{N-1}$, and $\mathcal{W}_{N}(t)$, named weight, is defined as

$$
\mathcal{W}_{N}(t)=\epsilon^{N} \int_{0}^{t} d s_{1} \int_{s_{1}}^{t} d s_{2} \ldots \int_{s_{N-1}}^{t} d s_{N} e^{-V_{0} s_{1}-V_{1}\left(s_{2}-s_{1}\right)-\ldots-V_{N}\left(t-s_{N}\right)} .
$$

\subsection{Evaluation of the weights}

According to their definition, the weights satisfy a recursive differential equation which is easily solved in terms of the Laplace transform $\widetilde{\mathcal{W}}_{N}(z)^{5}$, i.e.

$$
\widetilde{\mathcal{W}}_{N}(z)=\epsilon^{N} \prod_{V \in \mathscr{V}} \frac{1}{(z+V)^{N_{V}}}
$$


While this expression shows that $\mathcal{W}_{N}(t)$ depends on the multiplicities $N_{V}$ for any value of $N$, the explicit inversion of the Laplace transform can be done analytically only for $N$ large. However, this is the limit we are interested in since the weights $\mathcal{W}_{N}(t)$ have a maximum at some $N$ which increases by increasing $t$. By using a complex saddle-point method which is asymptotically exact for $N \rightarrow \infty$, we get ${ }^{5}$

$$
\mathcal{W}_{N}(t)=\frac{e^{x_{0} t-\sum_{V \in \mathscr{V}} N_{V} \log \left[\left(x_{0}+V\right) / \epsilon\right]}}{\sqrt{2 \pi \sum_{V \in \mathscr{V}} \frac{\epsilon^{2} N_{V}}{\left(x_{0}+V\right)^{2}}}}
$$

where $x_{0}$ is the solution of the equation

$$
\sum_{V \in \mathscr{V}} \frac{N_{V}}{x_{0}+V}=t
$$

\subsection{Canonical averages via a central limit theorem}

To evaluate the canonical averages it is useful to introduce the frequencies, $\nu_{V}=$ $N_{V} / N$ and $\nu_{A}=N_{A} / N$, which for $N$ large become continuously distributed in the range $[0,1]$ with the constraints

$$
\sum_{V \in \mathscr{V}} \nu_{V}=\sum_{A \in \mathscr{A}} \nu_{A}=1
$$

Note that for $N$ large we will not distinguish the different normalizations, $N+1$ and $N$, of $N_{V}$ and $N_{A}$, respectively. Equation (7) can be then rewritten as

$$
\left\langle\mathcal{W}_{N}(t) \prod_{A \in \mathscr{A}} A^{N_{A}}\right\rangle_{N}=\int d \boldsymbol{\nu} \mathcal{P}_{N}(\boldsymbol{\nu}) \frac{e^{x_{0} t+N(\boldsymbol{\nu}, \boldsymbol{u})}}{\sqrt{2 \pi N \sum_{V \in \mathscr{V}} \frac{\epsilon^{2} \nu_{V}}{\left(x_{0}+V\right)^{2}}}},
$$

where $\boldsymbol{\nu}$ and $\boldsymbol{u}$ are vectors with $m=m_{\mathscr{V}}+m_{\mathscr{A}}$ components defined as $\boldsymbol{\nu}^{T}=$ $\left(\ldots \nu_{V} \ldots ; \ldots \nu_{A} \ldots\right)$ and $\boldsymbol{u}^{T}=\left(\ldots-\log \left[\left(x_{0}+V\right) / \epsilon\right] \ldots ; \ldots \log A \ldots\right)$, respectively. For later use, we also define $\boldsymbol{v}^{T}=\left(\ldots\left(x_{0}+V\right)^{-1} \ldots ; \ldots 0 \ldots\right)$ and $\boldsymbol{w}^{T}=\left(\ldots\left(x_{0}+\right.\right.$ $\left.V)^{-2} \ldots ; \ldots 0 \ldots\right)$. Note that $\boldsymbol{u}, \boldsymbol{v}$ and $\boldsymbol{w}$ depend on $\boldsymbol{\nu}$ through $x_{0}=x_{0}(\boldsymbol{\nu})$ and $\boldsymbol{v}=-\partial_{x_{0}} \boldsymbol{u}, \boldsymbol{w}=-\partial_{x_{0}} \boldsymbol{v}$.

The probability density $\mathcal{P}_{N}(\boldsymbol{\nu})$ is given by the fraction of trajectories branching from the initial configuration $\boldsymbol{n}_{0}$ and having after $N$ jumps multiplicities $N_{V}=$ $\nu_{V} N$ and $N_{A}=\nu_{A} N$. For $N$ large, it can be approximated in the following way. We rewrite the multiplicities as $N_{V}=\sum_{k=0}^{N} \chi_{V}\left(\boldsymbol{n}_{k}\right)$ and $N_{A}=\sum_{k=1}^{N} \chi_{A}\left(\boldsymbol{n}_{k-1}\right)$, where $\chi_{V}(\boldsymbol{n})=1$ if $V(\boldsymbol{n})=V$ and $\chi_{V}(\boldsymbol{n})=0$ otherwise, and similarly for $\chi_{A}$. Since the configurations $\boldsymbol{n}_{k}$ form a Markov chain with finite state space, a central limit theorem applies to each rescaled sum $N_{V} / \sqrt{N}$ or $N_{A} / \sqrt{N}^{6}$. Therefore, these rescaled variables are completely described in terms of the mean values $\bar{\nu} \sqrt{N}$ and of the covariance matrix $\boldsymbol{\Sigma}$, which are easily measured by sampling over trajectories with a large number of jumps. Due to the constraints (12), it is easy to see that

$$
\sum_{V \in \mathscr{V}} \bar{\nu}_{V}=\sum_{A \in \mathscr{A}} \bar{\nu}_{A}=1
$$




$$
\sum_{V \in \mathscr{V}} \Sigma_{\alpha, V}=\sum_{V \in \mathscr{V}} \Sigma_{V, \alpha}=\sum_{A \in \mathscr{A}} \Sigma_{\alpha, A}=\sum_{A \in \mathscr{A}} \Sigma_{A, \alpha}=0, \quad \alpha \in \mathscr{A} \cup \mathscr{V} .
$$

In the next two subsections we will describe two different choices for the density $\mathcal{P}_{N}(\boldsymbol{\nu})$. The two densities differ only for the fact that the first one satisfies the constraints (12) in mean, while the other one identically. We will show that, at least in the limit of large $N$, the results obtained by assuming the two densities coincide.

\subsubsection{Purely Gaussian density}

In this case, we assume a purely Gaussian probability density

$$
\mathcal{P}_{N}(\boldsymbol{\nu})=\sqrt{\frac{N^{m}\left|\operatorname{det} \boldsymbol{\Sigma}^{-1}\right|}{(2 \pi)^{m}}} e^{-\frac{N}{2}\left(\boldsymbol{\Sigma}^{-1}(\boldsymbol{\nu}-\overline{\boldsymbol{\nu}}),(\boldsymbol{\nu}-\overline{\boldsymbol{\nu}})\right)} .
$$

Due to Eq. (15), we have that $\operatorname{det} \boldsymbol{\Sigma}=0$, i.e. $\boldsymbol{\Sigma}$ is singular and the density (16) ill defined. Nevertheless, this singularity is eliminable and the calculation with this density has the advantage to be rather simple. We rewrite Eq. (13) as

$$
\left\langle\mathcal{W}_{N}(t) \prod_{A \in \mathscr{A}} A^{N_{A}}\right\rangle_{N}=\int d \boldsymbol{\nu} e^{N \phi(\boldsymbol{\nu})} R(\boldsymbol{\nu})
$$

where

$$
\phi(\boldsymbol{\nu})=x_{0} \frac{t}{N}+(\boldsymbol{\nu}, \boldsymbol{u})-\frac{1}{2}\left(\boldsymbol{\Sigma}^{-1}(\boldsymbol{\nu}-\overline{\boldsymbol{\nu}}),(\boldsymbol{\nu}-\overline{\boldsymbol{\nu}})\right)
$$

and $R(\boldsymbol{\nu})$ is a smooth function. The parametric dependence of $\phi$ and $R$ on $t$ and $N$ is omitted for brevity. We will perform the integral (17) with a saddle-point method by solving the $m$-dimensional stationary problem for $\phi(\boldsymbol{\nu})$. The derivatives of $\phi$ with respect to the $V$ and $A$ components of $\boldsymbol{\nu}$ read

$$
\left\{\begin{array}{l}
\partial_{\nu_{V}} \phi(\boldsymbol{\nu})=\frac{t}{N} \partial_{\nu_{V}} x_{0}-(\boldsymbol{\nu}, \boldsymbol{v}) \partial_{\nu_{V}} x_{0}+u_{V}-\left(\boldsymbol{\Sigma}^{-1}(\boldsymbol{\nu}-\overline{\boldsymbol{\nu}})\right)_{V} \\
\partial_{\nu_{A}} \phi(\boldsymbol{\nu})=u_{A}-\left(\boldsymbol{\Sigma}^{-1}(\boldsymbol{\nu}-\overline{\boldsymbol{\nu}})\right)_{A} .
\end{array}\right.
$$

On the other hand, by using the definition of $x_{0}(\boldsymbol{\nu})$, Eq. (11), it is easy to see that $(\boldsymbol{\nu}, \boldsymbol{v})=t / N$, so that $\phi(\boldsymbol{\nu})$ is stationary for $\boldsymbol{\nu}=\boldsymbol{\nu}^{\mathrm{sp}}$ solution of the equation

$$
\boldsymbol{\nu}^{\mathrm{sp}}=\overline{\boldsymbol{\nu}}+\boldsymbol{\Sigma} \boldsymbol{u}\left(\boldsymbol{\nu}^{\mathrm{sp}}\right) .
$$

Note that, due to Eqs. (14) and (15), we have $\sum_{V \in \mathscr{V}} \nu_{V}^{\mathrm{sp}}=\sum_{A \in \mathscr{A}} \nu_{A}^{\mathrm{sp}}=1$ and only $m-2$ equations in (19) are independent. The result of the saddle-point integration then is

$$
\left\langle\mathcal{W}_{N}(t) \prod_{A \in \mathscr{A}} A^{N_{A}}\right\rangle_{N}=e^{N \phi\left(\boldsymbol{\nu}^{\mathrm{sp}}\right)} R\left(\boldsymbol{\nu}^{\mathrm{sp}}\right) \sqrt{\frac{(2 \pi)^{m}}{N^{m}\left|\operatorname{det} \nabla^{2} \phi\left(\boldsymbol{\nu}^{\mathrm{sp}}\right)\right|}},
$$

where

$$
\phi\left(\boldsymbol{\nu}^{\mathrm{sp}}\right)=\left[x_{0} \frac{t}{N}+(\overline{\boldsymbol{\nu}}, \boldsymbol{u})+\frac{1}{2}(\boldsymbol{\Sigma} \boldsymbol{u}, \boldsymbol{u})\right]_{\boldsymbol{\nu}=\boldsymbol{\nu}^{\mathrm{sp}}}
$$


and $\nabla^{2} \phi\left(\boldsymbol{\nu}^{\mathrm{sp}}\right)=-\boldsymbol{\Sigma}^{-1}-\boldsymbol{A}\left(\boldsymbol{\nu}^{\mathrm{sp}}\right), \boldsymbol{A}$ being the $m \times m$ matrix with elements

$$
A_{\alpha, \beta}=\frac{v_{\alpha} v_{\beta}}{(\boldsymbol{\nu}, \boldsymbol{w})}, \quad \alpha, \beta \in \mathscr{V} \cup \mathscr{A} .
$$

By writing $\operatorname{det} \nabla^{2} \phi\left(\boldsymbol{\nu}^{\mathrm{sp}}\right)=-\operatorname{det} \boldsymbol{\Sigma}^{-1} \operatorname{det}(\mathbf{1}+\boldsymbol{\Sigma} \boldsymbol{A})$ and observing that $\operatorname{det} \boldsymbol{\Sigma}^{-1}$ cancels out, Eq. (26) becomes

$$
\left\langle\mathcal{W}_{N}(t) \prod_{A \in \mathscr{A}} A^{N_{A}}\right\rangle_{N}=\left.\frac{1}{\sqrt{|\operatorname{det}(\mathbf{1}+\boldsymbol{\Sigma} \boldsymbol{A})|}} \frac{e^{x_{0} t+N\left[(\overline{\boldsymbol{\nu}}, \boldsymbol{u})+\frac{1}{2}(\boldsymbol{\Sigma} \boldsymbol{u}, \boldsymbol{u})\right]}}{\sqrt{2 \pi N \epsilon^{2}(\boldsymbol{\nu}, \boldsymbol{w})}}\right|_{\boldsymbol{\nu}=\boldsymbol{\nu}^{\mathrm{s} \mathrm{p}}} .
$$

Note that for $\hat{V} \equiv 0$ the matrix $\boldsymbol{A}$ is uniform and $\operatorname{det}(\mathbf{1}+\boldsymbol{\Sigma} \boldsymbol{A})=1$. In general, $\operatorname{det}(\boldsymbol{\Sigma} \boldsymbol{A})=0$ so that $\operatorname{det}(\mathbf{1}+\boldsymbol{\Sigma} \boldsymbol{A}) \simeq 1$ up to terms of second order in $\boldsymbol{A}$.

\subsubsection{Density with constraints identically satisfied}

In the previous subsection we have evaluated the canonical averages by exploiting a central limit theorem for the rescaled variables $N_{V} / \sqrt{N}$ and $N_{A} / \sqrt{N}$. However, due to the constraints (12), the joint probability for these $m$ rescaled sums is not purely Gaussian. Given an arbitrary set of $m_{\mathscr{V}}-1 V$-like components and $m_{\mathscr{A}}-1$ $A$-like components, we can assume a joint probability density as the product of a Gaussian density for this set of $m-2$ variables and two delta functions which take into account the constraints (12), i.e.

$$
\mathcal{P}_{N}(\boldsymbol{\nu})=\mathcal{F}_{N}(\hat{\boldsymbol{\nu}}) \delta\left(\sum_{V \in \mathscr{V}} \nu_{V}-1\right) \delta\left(\sum_{A \in \mathscr{A}} \nu_{A}-1\right),
$$

where $\mathcal{F}_{N}(\hat{\boldsymbol{\nu}})$ is the normal density defined in terms of the vector $\hat{\boldsymbol{\nu}}$ having the $m-2$ chosen components of $\boldsymbol{\nu}$

$$
\mathcal{F}_{N}(\hat{\boldsymbol{\nu}})=\sqrt{\frac{N^{m-2}\left|\operatorname{det} \hat{\boldsymbol{\Sigma}}^{-1}\right|}{(2 \pi)^{m-2}}} e^{-\frac{N}{2}\left(\hat{\boldsymbol{\Sigma}}^{-1}(\hat{\boldsymbol{\nu}}-\hat{\boldsymbol{\nu}}),(\hat{\boldsymbol{\nu}}-\hat{\bar{\nu}})\right)} .
$$

In the following, with the symbol ^ over a vector or a matrix we will indicate the projection onto the chosen $m-2$ dimensional index space.

By using the density (24), we rewrite Eq. (13) as

$$
\left\langle\mathcal{W}_{N}(t) \prod_{A \in \mathscr{A}} A^{N_{A}}\right\rangle_{N}=\int d \hat{\boldsymbol{\nu}} e^{N \hat{\phi}(\hat{\boldsymbol{\nu}})} \hat{R}(\hat{\boldsymbol{\nu}})
$$

where $\hat{R}$ is a smooth function of $\hat{\boldsymbol{\nu}}$ and, if $V_{*}$ and $A_{*}$ are the two components chosen to reduce to $m-2$ the dimension of the index space,

$$
\begin{aligned}
\hat{\phi}(\hat{\boldsymbol{\nu}})= & \hat{x}_{0} \frac{t}{N}+\left(\hat{\boldsymbol{\nu}}, \hat{\boldsymbol{u}}\left(\hat{x}_{0}\right)\right)+\left(1-\sum_{V \in \mathscr{V} \backslash V_{*}} \hat{\nu}_{V}\right) u_{V_{*}}\left(\hat{x}_{0}\right)+\left(1-\sum_{A \in \mathscr{A} \backslash A_{*}} \hat{\nu}_{A}\right) u_{A_{*}} \\
& -\frac{1}{2}\left(\hat{\boldsymbol{\Sigma}}^{-1}(\hat{\boldsymbol{\nu}}-\hat{\overline{\boldsymbol{\nu}}}),(\hat{\boldsymbol{\nu}}-\hat{\overline{\boldsymbol{\nu}}})\right) .
\end{aligned}
$$


Note that $\hat{x}_{0}=\hat{x}_{0}(\hat{\boldsymbol{\nu}})$ is the solution of Eq. (11) where the constraints (12) have been explicited. The function $\hat{\phi}$ can be rewritten as

$$
\hat{\phi}(\hat{\boldsymbol{\nu}})=\hat{x}_{0} \frac{t}{N}+(\hat{\boldsymbol{\nu}}, \hat{\delta \boldsymbol{u}})-\frac{1}{2}\left(\hat{\boldsymbol{\Sigma}}^{-1}(\hat{\boldsymbol{\nu}}-\hat{\overline{\boldsymbol{\nu}}}),(\hat{\boldsymbol{\nu}}-\hat{\boldsymbol{\nu}})\right)+u_{V_{*}}+u_{A_{*}},
$$

where $\delta \boldsymbol{u}=\boldsymbol{u}-\boldsymbol{u}_{0}$ and $\boldsymbol{u}_{0}^{T}=\left(u_{V_{*}} \ldots u_{V_{*}} ; u_{A_{*}} \ldots u_{A_{*}}\right)$. By evaluating the derivatives of $\hat{\phi}$ with respect to the components of $\hat{\boldsymbol{\nu}}$, we get

$$
\left\{\begin{array}{l}
\partial_{\hat{\nu}_{V}} \hat{\phi}(\hat{\boldsymbol{\nu}})=\frac{t}{N} \partial_{\hat{\nu}_{V}} \hat{x}_{0}-(\hat{\boldsymbol{\nu}}, \hat{\delta \boldsymbol{v}}) \partial_{\hat{\nu}_{V}} \hat{x}_{0}+\delta u_{V}-\left(\hat{\boldsymbol{\Sigma}}^{-1}(\hat{\boldsymbol{\nu}}-\hat{\overline{\boldsymbol{\nu}}})\right)_{V}-v_{V_{*}} \partial_{\hat{\nu}_{V}} \hat{x}_{0} \\
\partial_{\hat{\nu}_{A}} \hat{\phi}(\hat{\boldsymbol{\nu}})=\delta u_{A}-\left(\hat{\boldsymbol{\Sigma}}^{-1}(\hat{\boldsymbol{\nu}}-\hat{\boldsymbol{\nu}})\right)_{A},
\end{array}\right.
$$

where $\delta \boldsymbol{v}=-\partial_{\hat{x}_{0}} \delta \boldsymbol{u}=-\left(\partial_{\hat{x}_{0}} \boldsymbol{u}-\partial_{\hat{x}_{0}} \boldsymbol{u}_{0}\right)$. Observing that $(\hat{\boldsymbol{\nu}}, \hat{\delta \boldsymbol{v}})+v_{V_{*}}=t / N$, we can rewrite these derivatives as

$$
\left\{\begin{array}{l}
\partial_{\hat{\nu}_{V}} \hat{\phi}(\hat{\boldsymbol{\nu}})=\delta u_{V}-\left(\hat{\boldsymbol{\Sigma}}^{-1}(\hat{\boldsymbol{\nu}}-\hat{\overline{\boldsymbol{\nu}}})\right)_{V} \\
\partial_{\hat{\nu}_{A}} \hat{\phi}(\hat{\boldsymbol{\nu}})=\delta u_{A}-\left(\hat{\boldsymbol{\Sigma}}^{-1}(\hat{\boldsymbol{\nu}}-\hat{\boldsymbol{\nu}})\right)_{A}
\end{array}\right.
$$

Therefore, the saddle-point equation is

$$
\hat{\boldsymbol{\nu}}^{\mathrm{sp}}=\hat{\boldsymbol{\nu}}+\hat{\boldsymbol{\Sigma}} \hat{\delta \boldsymbol{u}}\left(\hat{\boldsymbol{\nu}}^{\mathrm{sp}}\right) .
$$

Finally, we calculate $\hat{\phi}\left(\hat{\boldsymbol{\nu}}^{\text {sp }}\right)$. By using Eqs. (28) and (29) and the identities $(\hat{\overline{\boldsymbol{\nu}}}, \hat{\delta \boldsymbol{u}})+$ $u_{V_{*}}+u_{A_{*}}=(\overline{\boldsymbol{\nu}}, \boldsymbol{u})$ and $(\hat{\boldsymbol{\Sigma}} \hat{\boldsymbol{u}}, \hat{\delta \boldsymbol{u}})=(\boldsymbol{\Sigma} \boldsymbol{u}, \boldsymbol{u})$, we get

$$
\begin{aligned}
\hat{\phi}\left(\hat{\boldsymbol{\nu}}^{\mathrm{sp}}\right) & =\left[\hat{x}_{0} \frac{t}{N}+(\hat{\overline{\boldsymbol{\nu}}}+\hat{\boldsymbol{\Sigma}} \hat{\delta \boldsymbol{u}}, \delta \hat{\boldsymbol{u}})-\frac{1}{2}(\hat{\boldsymbol{\Sigma}} \hat{\delta \boldsymbol{u}}, \hat{\delta \boldsymbol{u}})+u_{V_{*}}+u_{A_{*}}\right]_{\hat{\boldsymbol{\nu}}=\hat{\boldsymbol{\nu}}^{\mathrm{sp}}} \\
& =\left[\hat{x}_{0} \frac{t}{N}+\left(\overline{\boldsymbol{\nu}}, \boldsymbol{u}\left(\hat{x}_{0}\right)\right)+\frac{1}{2}\left(\boldsymbol{\Sigma} \boldsymbol{u}\left(\hat{x}_{0}\right), \boldsymbol{u}\left(\hat{x}_{0}\right)\right)\right]_{\hat{\boldsymbol{\nu}}=\hat{\boldsymbol{\nu}}^{\mathrm{sp}}} .
\end{aligned}
$$

Due to the identity $(\hat{\boldsymbol{\Sigma}} \hat{\delta \boldsymbol{u}}, \hat{\delta \boldsymbol{v}})=(\boldsymbol{\Sigma} \boldsymbol{u}, \boldsymbol{v})$, we have $\hat{x}_{0}\left(\hat{\boldsymbol{\nu}}^{\mathrm{sp}}\right)=x_{0}\left(\boldsymbol{\nu}^{\mathrm{sp}}\right)$, where $x_{0}\left(\boldsymbol{\nu}^{\mathrm{sp}}\right)$ is the value obtained with the purely Gaussian density. Therefore, Eq. (30) shows that, at least at the saddle-point level, the chosen probability density provides the same results obtained in the previous subsection.

\subsection{Resumming the canonical series}

In order to evaluate the expectation $\mathrm{E}\left(\mathcal{M}_{\boldsymbol{n}_{0}}^{t}\right)$ we need to resum the series (6). For $t \rightarrow \infty$, the sum can be substituted with the integral

$$
\mathrm{E}\left(\mathcal{M}_{\boldsymbol{n}_{0}}^{t}\right)=\frac{1}{\sqrt{|\operatorname{det}(\mathbf{1}+\boldsymbol{\Sigma} \boldsymbol{A})|}} \int d N \frac{e^{\psi(N)}}{\sqrt{2 \pi N \epsilon^{2}\left(\boldsymbol{\nu}^{\mathrm{sp}}, \boldsymbol{w}\left(x_{0}\left(\boldsymbol{\nu}^{\mathrm{sp}}\right)\right)\right)}},
$$

where

$$
\psi(N)=x_{0}\left(\boldsymbol{\nu}^{\mathrm{sp}}\right) t+N\left[\left(\overline{\boldsymbol{\nu}}, \boldsymbol{u}\left(x_{0}\left(\boldsymbol{\nu}^{\mathrm{sp}}\right)\right)\right)+\frac{1}{2}\left(\boldsymbol{\Sigma} \boldsymbol{u}\left(x_{0}\left(\boldsymbol{\nu}^{\mathrm{sp}}\right)\right), \boldsymbol{u}\left(x_{0}\left(\boldsymbol{\nu}^{\mathrm{sp}}\right)\right)\right)\right] .
$$


The integrand in (31) is exponentially peaked at $N=N^{\text {sp }}$, where $N^{\text {sp }}$ satisfies $\partial_{N} \psi\left(N^{\text {sp }}\right)=0$. For a generic $N$ we have

$$
\begin{aligned}
\partial_{N} \psi(N) & =\left[\{t-N[(\overline{\boldsymbol{\nu}}, \boldsymbol{v})+(\boldsymbol{\Sigma} \boldsymbol{u}, \boldsymbol{v})]\} \partial_{N} x_{0}+(\overline{\boldsymbol{\nu}}, \boldsymbol{u})+\frac{1}{2}(\boldsymbol{\Sigma} \boldsymbol{u}, \boldsymbol{u})\right]_{\boldsymbol{\nu}=\boldsymbol{\nu}^{\mathrm{sp}}} \\
& =\left[(\overline{\boldsymbol{\nu}}, \boldsymbol{u})+\frac{1}{2}(\boldsymbol{\Sigma} \boldsymbol{u}, \boldsymbol{u})\right]_{\boldsymbol{\nu}=\boldsymbol{\nu}^{\mathrm{sp}}},
\end{aligned}
$$

where, due to Eqs. (11) and (19), we noticed that the term $\{t-N[(\overline{\boldsymbol{\nu}}, \boldsymbol{v})+(\boldsymbol{\Sigma} \boldsymbol{u}, \boldsymbol{v})]\}$ vanishes for $\boldsymbol{\nu}=\boldsymbol{\nu}^{\mathrm{sp}}$. The stationarity condition for $\psi(N)$ is, therefore,

$$
\left[(\bar{\nu}, \boldsymbol{u})+\frac{1}{2}(\boldsymbol{\Sigma} \boldsymbol{u}, \boldsymbol{u})\right]_{\boldsymbol{\nu}=\boldsymbol{\nu}^{\mathrm{sp}}, N=N^{\mathrm{sp}}}=0 .
$$

Equation (33) is a time independent equation which determines $\left.x_{0}\right|_{\boldsymbol{\nu}=\boldsymbol{\nu}^{\mathrm{sp}}, N=N^{\mathrm{sp}}}$ as a function of $\overline{\boldsymbol{\nu}}$ and $\boldsymbol{\Sigma}$. According to Eq. (11), this means that the quantity $N^{\text {sp }}$ increases linearly with $t$ so that $\left.x_{0}\right|_{\boldsymbol{\nu}=\boldsymbol{\nu}^{\mathrm{sp}}, N=N^{\text {sp }}}$ becomes independent of time in the limit $t \rightarrow \infty$. By evaluating the second derivative of $\psi(N)$

$$
\partial_{N}^{2} \psi(N)=-\left(\boldsymbol{\nu}^{\mathrm{sp}}, \boldsymbol{v}\left(x_{0}\left(\boldsymbol{\nu}^{\mathrm{sp}}\right)\right)\right) \partial_{N} x_{0}\left(\boldsymbol{\nu}^{\mathrm{sp}}\right),
$$

where

$$
\partial_{N} x_{0}\left(\boldsymbol{\nu}^{\mathrm{sp}}\right)=\frac{1}{N} \frac{\left(\boldsymbol{\nu}^{\mathrm{sp}}, \boldsymbol{v}\left(x_{0}\left(\boldsymbol{\nu}^{\mathrm{sp}}\right)\right)\right)}{\left(\boldsymbol{\nu}^{\mathrm{sp}}, \boldsymbol{w}\left(x_{0}\left(\boldsymbol{\nu}^{\mathrm{sp}}\right)\right)\right)+\left(\boldsymbol{v}\left(x_{0}\left(\boldsymbol{\nu}^{\mathrm{sp}}\right)\right), \boldsymbol{\Sigma} \boldsymbol{v}\left(x_{0}\left(\boldsymbol{\nu}^{\mathrm{sp}}\right)\right)\right)},
$$

and approximating $\psi(N) \simeq \psi\left(N^{\mathrm{sp}}\right)+\frac{1}{2} \partial_{N}^{2} \psi\left(N^{\mathrm{sp}}\right)\left(N-N^{\mathrm{sp}}\right)^{2}$ in the exponent and $N \simeq N^{\text {sp }}$ elsewhere, the integral (31) reduces to a Gaussian one which gives

$$
\mathrm{E}\left(\mathcal{M}_{\boldsymbol{n}_{0}}^{t}\right)=\left.\sqrt{\frac{1+\operatorname{tr}(\boldsymbol{\Sigma} \boldsymbol{A})}{|\operatorname{det}(\mathbf{1}+\boldsymbol{\Sigma} \boldsymbol{A})|}} \frac{e^{x_{0} t}}{\epsilon(\boldsymbol{\nu}, \boldsymbol{v})}\right|_{\boldsymbol{\nu}=\boldsymbol{\nu}^{\mathrm{sp}}, N=N^{\mathrm{sp}}} .
$$

Note that, since the peak of the Gaussian at $N=N^{\text {sp }}$ moves to infinity linearly with $t$ while its width increases only as $\sqrt{t}$, the result (36) is asymptotically exact for $t \rightarrow \infty$.

According to Eq. (2), the result (36) shows that the ground-state energy of the hard-core boson system is

$$
E_{0 B}=-\left.x_{0}\right|_{\nu=\nu^{\mathrm{sp}}, N=N^{\mathrm{sp}}} .
$$

Equation (33) is, therefore, the equation for the ground-state energy. It defines $E_{0 B}$ in terms of $\bar{\nu}$ and $\boldsymbol{\Sigma}$ and explicitly reads

$$
\begin{aligned}
0= & -\sum_{V \in \mathscr{V}} \bar{\nu}_{V} \log \left(\frac{-E_{0 B}+V}{\epsilon}\right)+\sum_{A \in \mathscr{A}} \bar{\nu}_{A} \log (A) \\
& +\frac{1}{2} \sum_{V \in \mathscr{V}} \sum_{V^{\prime} \in \mathscr{V}} \Sigma_{V, V^{\prime}} \log \left(\frac{-E_{0 B}+V}{\epsilon}\right) \log \left(\frac{-E_{0 B}+V^{\prime}}{\epsilon}\right) \\
& -\sum_{V \in \mathscr{V}} \sum_{A \in \mathscr{A}} \Sigma_{V, A} \log \left(\frac{-E_{0 B}+V}{\epsilon}\right) \log (A)
\end{aligned}
$$




$$
+\frac{1}{2} \sum_{A \in \mathscr{A}} \sum_{A^{\prime} \in \mathscr{A}} \Sigma_{A, A^{\prime}} \log (A) \log \left(A^{\prime}\right)
$$

In the case $\hat{V} \equiv 0$, the ground-state energy $E_{0 B}^{(0)}$ can be solved analytically

$$
E_{0 B}^{(0)}=-\epsilon \exp \left[\sum_{A \in \mathscr{A}} \bar{\nu}_{A} \log (A)+\frac{1}{2} \sum_{A \in \mathscr{A}} \sum_{A^{\prime} \in \mathscr{A}} \Sigma_{A, A^{\prime}} \log (A) \log \left(A^{\prime}\right)\right] .
$$

\section{Conclusions}

By using saddle-point techniques and a central limit theorem, we have exploited an exact probabilistic representation of the quantum dynamics in a lattice to derive analytical approximations for the matrix elements of the evolution operator of a system of hard-core bosons in the limit of long times. The approach yields a simple scalar equation for the ground-state energy. This equation depends on the values of the generalized potentials $V$ and of the kinetic quantities $A$, and on the statistical moments $\overline{\boldsymbol{\nu}}$ and $\boldsymbol{\Sigma}$ of their asymptotic multiplicities $N_{V}$ and $N_{A}$. In turn, these moments depend only on the structure of the system Hamiltonian, not on the values of the Hamiltonian parameters. This implies that the statistical moments must be measured una tantum for a given Hamiltonian structure and, once $\overline{\boldsymbol{\nu}}$ and $\boldsymbol{\Sigma}$ are known, our approach provides the ground-state energy analytically as a function of the Hamiltonian parameters.

The ground state energies obtained with the present formula reveal a small systematic error when compared with exact results ${ }^{5}$. In fact, a more accurate analysis which takes into account the large deviations neglected by the central limit theorem shows that the present approach corresponds to a second order truncation of an exact cumulant expansion for the ground-state energy ${ }^{7}$.

Similar results hold in the case of Hamiltonians with arbitrary kinetic operators.

\section{References}

1. G. F. De Angelis, G. Jona-Lasinio and M. Sirugue, J. Phys. A 16, 2433 (1983).

2. G. F. De Angelis, G. Jona-Lasinio and V. Sidoravicius, J. Phys. A 31, 289 (1998).

3. M. Beccaria, C. Presilla, G. F. De Angelis and G. Jona Lasinio, Europhys. Lett. 48, 243 (1999).

4. S. Sorella and L. Capriotti, Phys. Rev. B 61, 2599 (2000).

5. M. Ostilli and C. Presilla, New J. Phys. 6, 107 (2004).

6. P. Billingsley, Probability and Measure (John Wiley \& Sons, New York, 1986).

7. M. Ostilli and C. Presilla, in preparation. 